\title{
MPEG-7 based Service Guide for Mobile TV*
}

\author{
Emin Gencpinar, Tolga Berber, Gamze Seckin, Adil Alpkocak \\ Dokuz Eylul University, Department of Computer Engineering, Izmir, Turkey \\ Vidiator Technology (US) Inc, WA, USA \\ emin.gencpinar@ogr.deu.edu.tr, \{tberber, alpkocak\}@cs.deu.edu.tr, gamzes@vidiator.com
}

\begin{abstract}
In this paper, we propose MPEG-7 based Electronic Service Guide (ESG) within Multimedia Broadcast Multicast System (MBMS). Our prototype covers OMA BCAST ESG fragments defined for MBMS and extends content fragment of ESG by MPEG-7. In order to demonstrate the usefulness of our approach, we implement a test application that provides a multimedia query for MBMS services and sessions, and retrieve a tree view of available services and a categorized view according to the genre grouping criteria.
\end{abstract}

\section{Keywords}

ESG, MBMS, MPEG-7, Mobile TV, Multimedia Query

\section{INTRODUCTION}

Wireless multimedia distribution has become very popular with the availability of new technologies. People want to capture photos and videos, store and share them easily with their digital mobile phones. Pervasive and ubiquitous computing affected multimedia devices to become smarter, smaller and mobile. Today both streaming and downloading services are offered over point-to-point wireless connections. Large scale distribution of media makes this point-to-point approach inefficient especially for wireless networks. The recent development in multimedia applications with a parallel progress in wireless transport technologies has brought real-time and non-real time multimedia distribution in the form of multicasting and broadcasting. Such multimedia distribution mechanisms include streaming and downloading services for on-demand video, mobile TV, short clips for news, football results, software updates and more.

Several technologies that provide broadcasting and multicasting for wireless networks are 3GPP MBMS (Multimedia Broadcast and Multicast System) [19] [2], 3GPP2 BCMCS (Broadcast and Multicast System), DVB-H (Digital Video Broadcast for Handhelds) that takes an advantage of high bearer rate network, and MediaFLO among others [11]. MBMS is a multicast and broadcast distribution technology for Third Generation (3G) Universal Mobile Telecommunications System (UMTS) wireless networks [16]. MBMS enables point-to-multipoint transmission of multimedia data by using the existing wireless networks with MBMS specific transmission procedures. MBMS

Permission to make digital or hard copies of all or part of this work for personal or classroom use is granted without fee provided that copies are not made or distributed for profit or commercial advantage and that copies bear this notice and the full citation on the first page. To copy otherwise, or republish, to post on servers or to redistribute to lists, requires prior specific permission and/or a fee.

MOBIMEDIA 2007, August 27-29, Nafpaktos, Greece

Copyright $\odot 2007$ ICST 978-963-06-2670-5

DOI 10.4108/ICST.MOBIMEDIA2007.1820 can work with low bearer rates, so consumes less resource allocation and bandwidth.

The main reasons behind the increasing interest in the broadcast and multicast service distribution is the independency from the number of users as well as the resource savings unlike unicast (i.e. point-to-point) packet switched streaming (PSS) for the same transmission power range. In other words in MBMS the number of concurrent users may be limited only by the base station maximum capacity.

There are two delivery modes defined within MBMS, the download and the streaming delivery mode. Download delivery mode is for delivering discrete objects like files where transmission reliability is important. Streaming delivery mode is for delivery of continuous media. For streaming, the video quality is important, but reliability is not compulsory. For either mode there is a need for service discovery or announcement, or a service guide mechanism [13]. A detailed view of MBMS system is given in [16] and [17].

Content description is a critical component of service offering. Service Guide (SG)'s are used by content providers to describe the services, how to access those services and content they make available for offering subscription or purchase of an item over broadcast or interaction channel. SG's are user entry points to discover the currently available or scheduled services and content. SG needs to be refreshed periodically to make functionality consistent [13]. ESG means that SG is electronically available on digital form. EPG (Electronic Program Guide) on the other hand is on screen program guide first used as cable TV guide. Today it is also used by satellite TV applications. Twenty four hour program guides are carried by TV guide channels. EPG data is used with a graphical user interface to view some content descriptions like program titles, start and end times, categorization of services according to channel or genre grouping. ESG covers EPG, because it describes how to access the services, how to purchase or subscribe to items.

The ISO/IEC Motion Picture Group (MPEG) issued in 2002 a standard, called MPEG-7, which enables the content description of multimedia data in XML. The standard supports applications to exchange, identify, and filter multimedia contents based on MPEG-7 descriptions.

In this paper, we propose to extend the broadcasting system in MBMS platform and use MPEG-7 to apply content semantics to the services. In order to demonstrate the usefulness of the new broadcasting environment, we implement a test application in MBMS platform over the UMTS network emulation, and experiment with two cases including real-time content filtering and content-based retrieval. The test application covers Open Mobile Alliance (OMA) Broadcast (BCAST) ESG [13]

\footnotetext{
"This work is part of EEEAG 104E163, a project sponsored by Vidiator Technology (US) Inc, TUBITAK, The Turkish National Science Foundation,
} and Dokuz Eylul University, Department of Computer Engineering. 
fragments defined for MBMS. Moreover, the content fragment of ESG is pruned and grafted into MPEG-7. It should be concluded that the proposed system and services offer an extended broadcasting environment which is more user-friendly and provides greater features for multimedia consumers.

Rest of this paper is organized as follows: In section II, related work is summarized. Section III provides the system architecture of MBMS and discusses MPEG-7 based ESG content fragments. Section IV describes the details of test application. The last section concludes the paper and gives a look to the future studies on this subject.

\section{RELATED WORK}

The work of Hoyeon Jang [5] is about Integrated Electronic Service Guide (IESG) which is designed to compose ESG, interactive TV service information. The EPG concept is expanded to IESG. The channel program schedule is received over the air, the information of other channels and the genre are processed using a return channel. This work focuses on Advanced Common Application Platform (ACAP) software which is a Java based data broadcasting standard for interactive TV service through digital TV set-top boxes. This work covers unresolved issues for providing EPG service using Advanced Television System Committee (ATSC) Program and System Information Protocol (PSIP).

The work of Jihye Lyu et.al. [7] is about design of open APIs for Personalized IPTV that is a broadcasting service enabling support of interactive and personalized services using IP network. This study extends the Parlay X Web service structure for the personalized EPG, Network Data Recorder (NDR) and Target Advertisement services. The work covers personalized EPG and focuses on the personalized services.

In a recent study [14], Sung Ho Jin et.al. proposed an intelligent broadcasting system for enhanced personalized services, based on the semantics of the broadcasting content. In this study they used the MPEG-7 and TV-Anytime Forum (TVAF), as well as an agent technology. For content-level services real-time content filtering, personalized video skimming, and contentbased retrieval using audio characteristic are implemented.

Andrea Kofler-Vogt et.al. in their study [1] pointed out that multimedia delivery especially in mobile applications that deal with MPEG-7 suffer from limited bandwidth, low computational power, and limited battery life. So, they described an index system adopted from database systems that allow filter mechanisms and random access to encoded MPEG7 streams and which overcome the limitation of the network and the consuming terminal. Encoding is applied in order to reduce the data rate of the XML documents to be transmitted. However, since MPEG-7 is a generic standard, not all components of MPEG-7 are necessary [8] for mobile applications. Even, a small subset of MPEG-7 can meet basic requirements of mobile applications.

INSTINCT is a European project converging DVB-CBMS (DVB Convergence of Broadcast and Mobile Services) activities considering DVB-T (DVB Terrestrial), DVB-H, DVB-MHP (DVB Multimedia Home Platform). INSTINCT partners Brunel, FTR\&D, Motorola and Netikos proposed an ESG data model which has been built over the skeleton of TV Anytime (TVA). That is, the ESG model is based on TVA and
XML, uses MPEG-7 syntax to separate the content descriptions from the delivery instances. However, the proposed ESG introduces new features for IP Datacasting over DVB-H. DVBCBMS ESG model would be probably a compromise between TVA, Nokia and INSTINCT proposals while INSTINCT proposal is well perceived by DVB-CBMS group. [9]

XML based ESG for mobile TV, EXPWAY's FastESG conforms mobile TV standards defined by DVB-CBMS, DVBIPI (DVB Internet Protocol Infrastructure), TVA, W3C and MPEG groups, provides multimedia search engines, uses BiM [10], an accepted binary XML transport format by MPEG-7, TVA, ARIB and DVB standards [4]. EXPWAY uses the BiM compression method for audiovisual metadata. BiM has been adopted by the ARIB and TVA, and recently by DVB GBS (DVB Generic Data Broadcasting) and DVB CBMS for compression of EPGs and ESGs [15].

The IP Datacast over DVB-H integrates ESG which is based on MPEG-7 [3] and TVA like existing standards. DVB Document A112 [6] discusses recommended restrictions on the use of imported MPEG-7 and TVA datatypes within the ESG data model.

\section{SYSTEM ARCHITECTURE}

MBMS user services involve protocols, codecs and delivery method procedures as shown in Figure 1, which only covers protocol stack of streaming delivery mode in MBMS protocol stack.

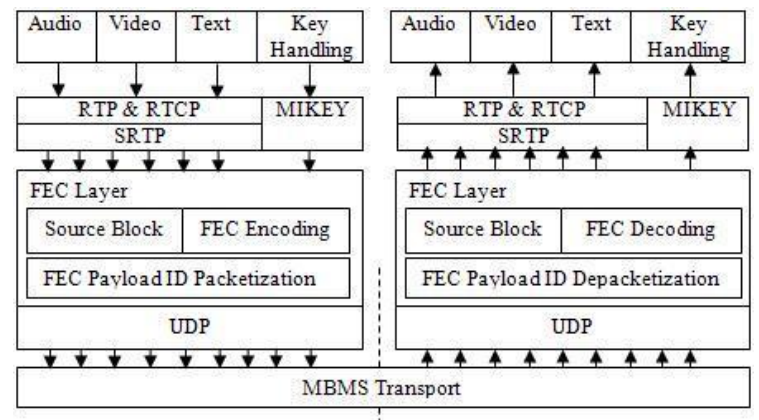

Figure 1. MBMS stack for streaming services [16]

OMA BCAST offers ESG architecture for MBMS. OMA BCAST defines Service Guide Delivery (SGD) fragments which are Service, Schedule, Content, and Access respectively. First three fragments obtain core of ESG structure and Access fragment covers session details that also includes some part of the Session Description Procedure (SDP) file. ESG also relates core fragments by Purchase Item. Purchase Item, Data and Channel are provisioning fragments. ESG structure has provisioning, core and access components as shown in Figure 2.

Figure 3 shows Vidiator's system model with OMA ESG. In this model, MBMS data is delivered through RTP (Real-time Transport Protocol) and FLUTE (File Delivery Over Unidirectional Transport) protocols, for streaming and downloading delivery modes, respectively. 


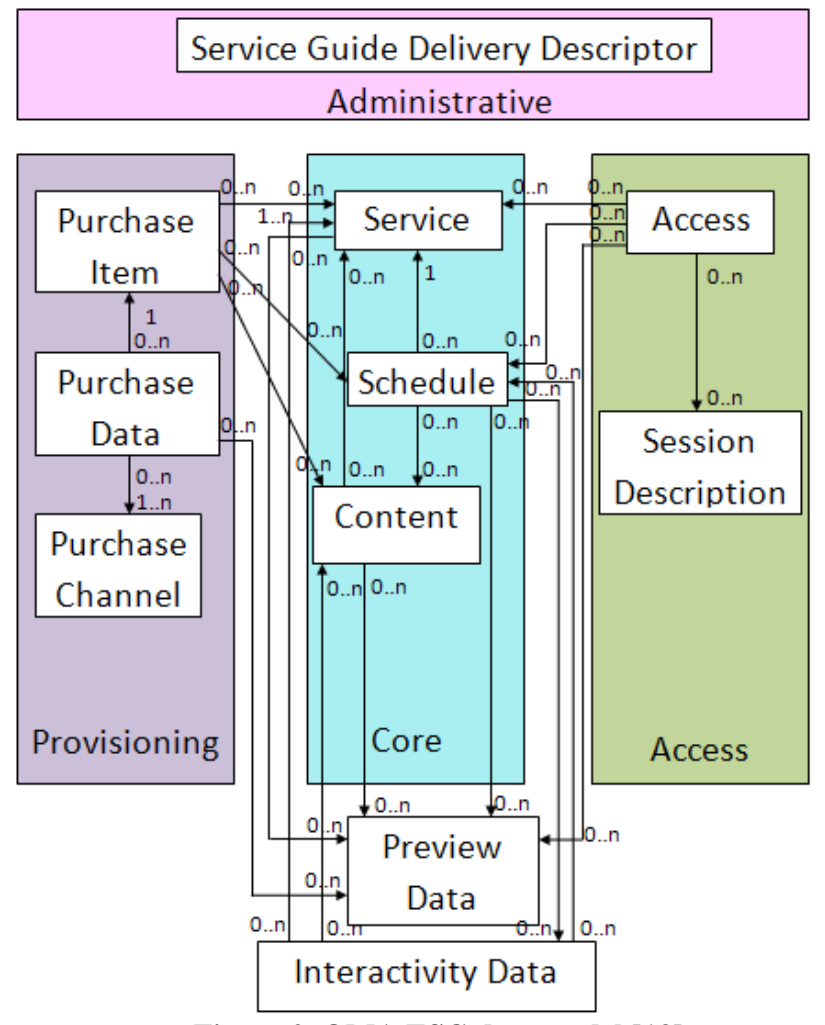

Figure 2. OMA ESG data model [13]

As operators create a new service, OMA BCAST ESG files are created. Vidiator's Xenon Live Encoder encodes the media content. MBMS Broadcast Multicast Service Centre (BM-SC) uses MBMS delivery functions to transmit media and OMA BCAST ESG fragments to User Equipments (UE). The MBMS receivers download ESG meta files for accessing the relevant sessions. ESG and MPEG-7 meta files are transmitted over FLUTE protocol. Sessions are bundled groups of services in ESG. Several sessions are grouped according to the timing and genre criteria in the content server. Each criterion can be considered as a service. Service Guide Delivery Descriptor in administrative section is a root meta file that refers ESG fragment meta files.

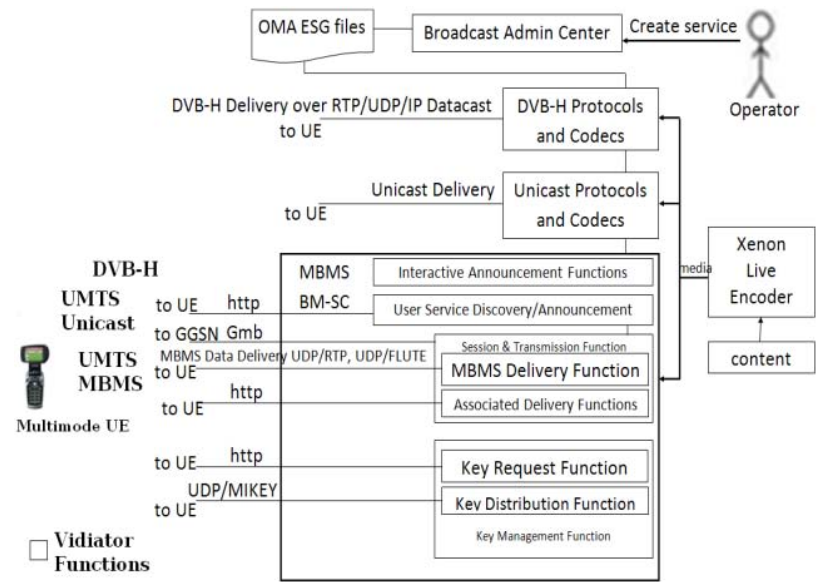

Figure 3. Vidiator system model with OMA ESG

\subsection{Extending MPEG-7}

MPEG-7 is a multimedia description standard, and endeavor to describe multimedia content in both high level and low level.
High level description components of MPEG-7 aim to point out semantic relationships between objects in multimedia content. On the other hand, low level descriptors can be extracted automatically from multimedia content. Besides, some annotation tools for MPEG-7 could be used to annotate multimedia objects manually (i.e. specifying keywords for multimedia content).

In this study, instead of associating MPEG-7 descriptions into ESG, we propose a different approach which extends MPEG-7 to be able to hold some service related information such as scheduling, parental rating etc.

Although annotation tools for MPEG-7 are sufficient to annotate multimedia content, MPEG uses XML schema, and allows MPEG-7 to extend when needed. There are two major ways to extend MPEG-7, which are DType and DSType. Figure 4 and Figure 5 show DType and DSType in XML schema fragments. Figure 6 depicts XSD schema of the Modified Text Annotation Type of MPEG-7, and Figure 10 gives a detailed view of the whole Mobile TV architecture, which is extended MPEG-7 Meta Structure, in class diagram format.

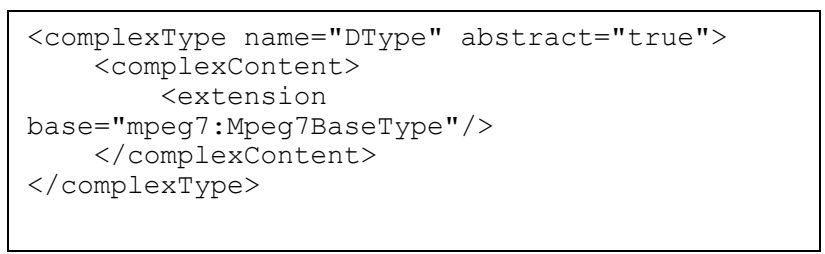

Figure 4. XSD schema of the DType

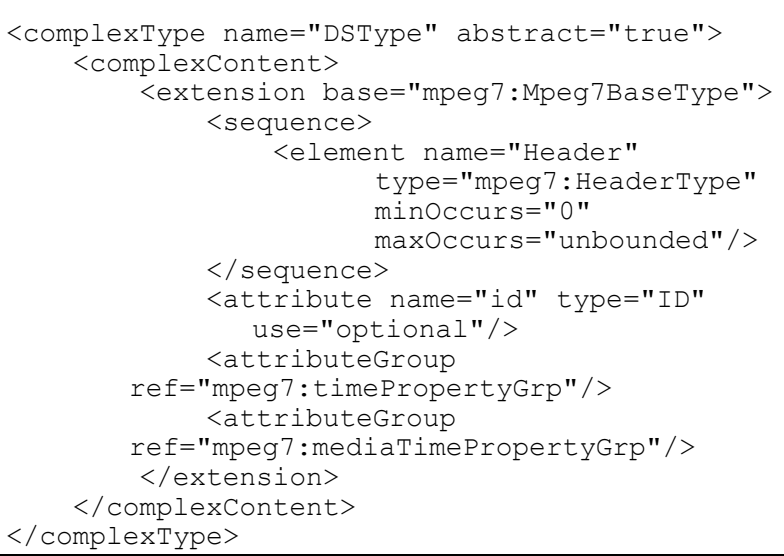

Figure 5. XSD schema of the DSType

\section{TEST APPLICATION}

In order to evaluate our approach we have developed a test application in Visual Studio .NET 2005 Smartphone Emulator, and a general view of application can be seen in Figure 7.

Test Application uses Vidiator's MBMS Xenon Live Encoder [18], Xenon Service Manager and Xenon Streamer as shown in Figure 8. Xenon Live Encoder, can encode both live and on demand video content in real-time and pass it to a streaming server for delivery. Xenon Live Encoder is being used by mobile operators and content providers for mobile multimedia services. MBMS Xenon Service Manager serves to create a new MBMS service. A service encapsulates delivery method parameters. Xenon Service Manager also shows existing MBMS services. 


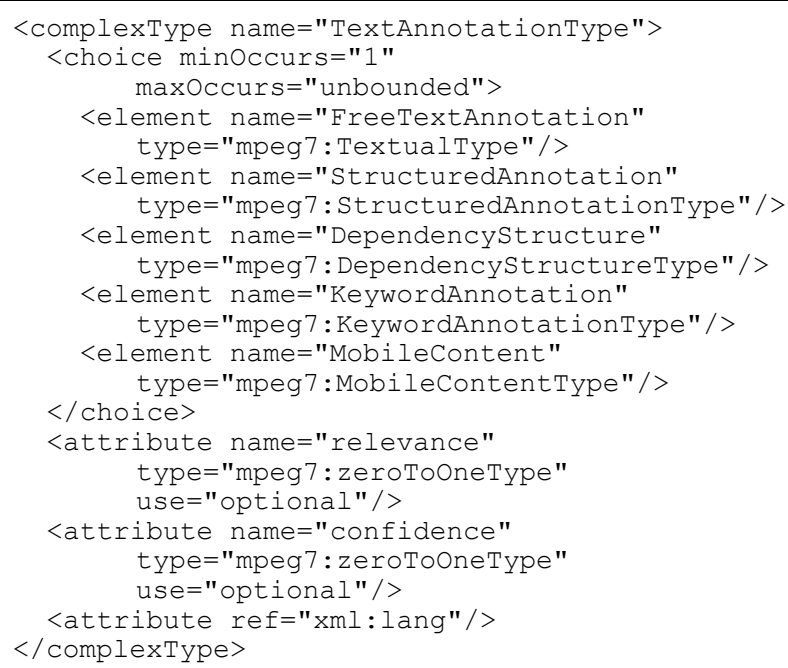

Figure 6. XSD schema of the modified text annotation type of MPEG-7

Xenon Streamer is an MBMS streaming server. It is used to multicast or to broadcast the active streaming services. Xenon Streamer is connected to Xenon Live Encoder by Smart Live Session Protocol (SLSP) which is a Vidiator's protocol for communicating with Xenon Live Encoder.

Vidiator's MBMS FLUTE Download Server is used for MBMS download and announcement sessions that announce an occurrence of a new user service by listing service details. The announcement service is a download service involving meta files sent to MBMS subscribers. OMA ESG [13] is also provided for session announcement. A subscriber joins any one of the valid services through the Service Guide Receiver.

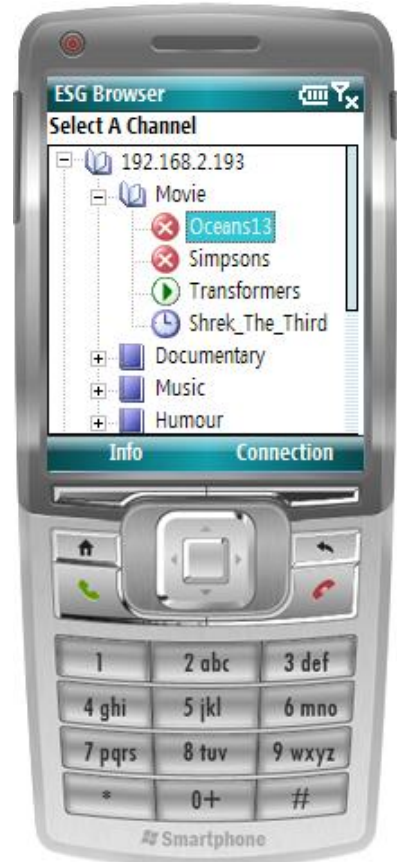

Figure 7. Appearance of service guide test application.

In order to emulate wireless network environment, we used NetEm [12], which is simple to use and provides variable delay, packet loss, duplication and reordering. It is a protocol independent emulator, and Bandwidth can be limited by a token bucket filter. UTRAN streaming simulation conditions and results, explained in 3GPP document [17], are leveraged to generate real network conditions.

The Service Guide Receiver (SGR) for mobile TV has been redesigned as shown in Figure 8, by extending MPEG-7. The SGR application has an iconized tree view of services, which are grouped according to genre under the content server source IP address. Iconized view also makes it easy to distinguish status of services as expired, streaming and scheduled services with different icons. Multimedia query interface, service details and its preview are shown in Figure 9-a, 9b and 9-c, respectively.

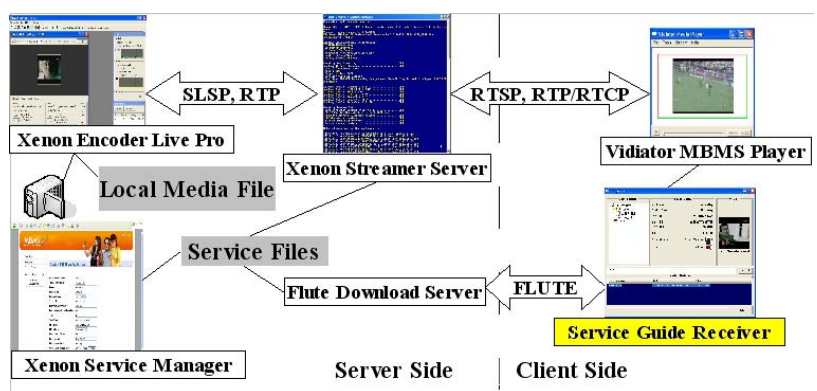

Figure 8. Vidiator MBMS system modules

The test application is also capable to perform simple multimedia query based on extended MPEG-7 that involves the content fragment in the core of ESG as in Figure 2. Multimedia query processor searches all the ESG files and MPEG-7 content descriptions. The query result grabs the service name, service genre by matching service to its location in a tree view structure and its status. If there is more than one possible result, these details make easier to assess the query result, as shown in Figure 9-a. By selecting within the tree view service names or selecting one of the query results, a user can connect a streaming video service by connect service button.

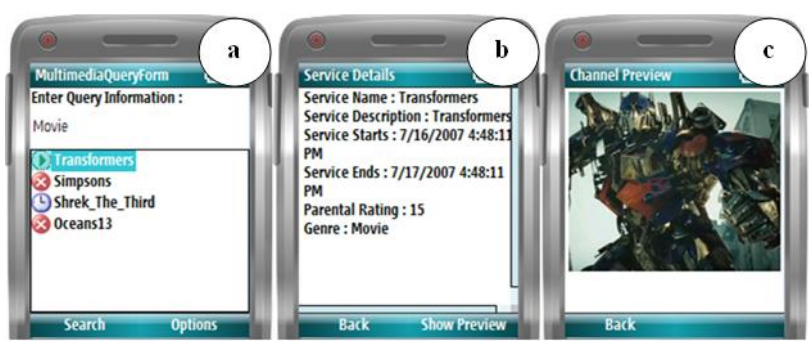

Figure 9. Service guide receiver with multimedia query.

We have observed that with the integration of MPEG-7 the system performance and complexity were not impacted. The query response time has been very low. MPEG-7 has provided value-added features for a better user service guide usage.

\section{CONCLUSION AND FUTURE WORK}

In this study we presented we propose MPEG-7 based Electronic Service Guide (ESG) based on Vidiator's MBMS architecture for Mobile TV applications. Our prototype covers OMA BCAST ESG fragments defined for MBMS and extends content fragment of ESG by MPEG-7.

We extended the existing OMA BCAST ESG with MPEG-7 meta data structure to provide a tree view and a genre based 
view of available services, and provided information on how service guide descriptions are addressed.

We have observed that the system performance and complexity were not impacted with the introduction of MPEG-7 meta data and the ESG experience has significantly improved with the addition of search feature which introduces negligible delays.

MPEG-7 contains a rich set of content description interfaces for multimedia data. However, limited resources of mobile environment force us to use a reduced version of MPEG-7. This is quite acceptable because MPEG-7 is a generic standard, not all components of MPEG-7 are necessary for mobile applications. In this study we have used only some of the manual annotations. However different description tools of MPEG-7, such as low level and some of the other semantic content descriptors can be used for mobile TV environment.

\section{REFERENCES}

[1] Andrea Kofler-Vogt, Harald Kosch, Joerg Heuer, Andre Kaup, BeTrIS-An Index System for MPEG-7 Streams, EURASIP Journal on Applied Signal Processing Article ID 15482, Pages 1-11, 2006.

[2] Broadcast Distribution System Adaptation 3GPP/MBMSDraft Version 1.0 - 19 Apr 2006, OMATS-BCAST_MBMS_Adaptation-V1_0-20060419-D

[3] ETSI TS 102471 V1.2.1, IP Datacast over DVB-H: Electronic Service Guide, November 2006

[4] Expway Launches Turnkey FastESG, Seamless Electronic Service Guide for Mobile TV, http://news.thomasnet.com/fullstory/468728/2585, October 2005

[5] Hoyeon Jang, Nammee Moon, Xlet-based, IESG (Integrated Electronic Service Guide) in ATSC-ACAP, Proc. of 4th International Conference on Software Engineering Research, Management and Applications SERA '06, Seoul, 2006
[6] IPDC over DVB-H ESG Implementation Guidelines, DVB Document A112, March 2007

[7] Jihye Lyu, Shinjee Pyo, Jeongyeon Lim, Munchurl Kim, Sunhwan Lim, Sangki Kim, Design of Open APIs for Personalized IPTV Service, Laboratory for Multimedia Computing, Communications, and Broadcasting, Broadband Convergence Network Division ETRI, February 2007.

[8] José M. Martínez, Rob Koenen, Fernando Pereira, MPEG-7: the generic Multimedia Content Description Standard, IEEE Multimedia, pp. 78 87, April-June 2002.

[9] M. Gross, J. Lauterjung, T. Levesque, B. Mazieres, Update on Deliverable 1.1, INSTINCT Project, March 2005

[10] Mike Cokus, MITRE Corporation Santiago PericasGeertsen, Sun Microsystems, XML Binary Characterization Use Cases, W3C Working Group Note, March 2005

[11] Mobile TV: The Groundbreaking Dimension v2.21, Mobile TV UMTSF/GSMA Joint Work Group Report, N.

[12] Netem (Network Emulator), http://linuxnet.osdl.org/index.php/Netem, November 2006.

[13] Service Guide for Mobile Broadcast Services Draft Version $1.0-24$ Mar 2006, OMA-TSBCAST_ServiceGuide-V1_0_0-20060324-D

[14] Sung Ho Jin, Tae Meon Bae, Yong Man Ro, Hoi-Rin Kim, Munchurl Kim, Intelligent broadcasting system and services for personalized semantic contents consumption, Expert Systems with Applications Vol. 31, pp. 164-173, 2006.

[15] The BiM compression method for XML Data, Expway

[16] 3GPP TS 26.346 V7.2.0 (2006-12), MBMS Protocols and Codecs, December 2006.

[17] 3GPP TR 26.946 V6.1.0 (2006-09), MBMS User Service Guidelines, September 2006.

[18] www.vidiator.com

[19] http://www.3gpp.org 


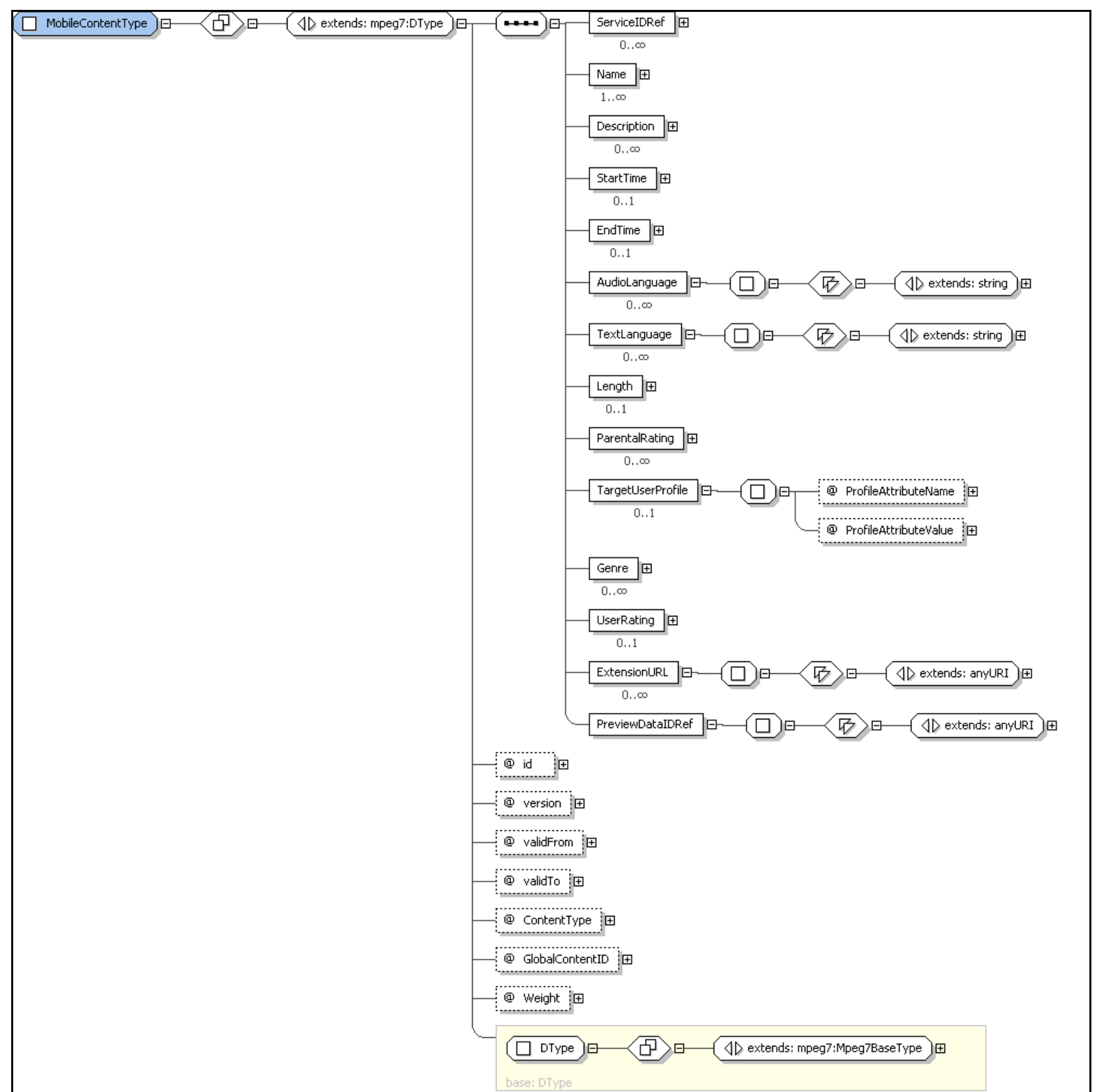

Figure 10. Class diagram of Mobile TV extended MPEG-7 meta structure. 